\title{
Mechanisms of Deformation Twinning Near Crack Tips in Nanostructured Materials
}

\author{
N.V.Skiba \\ Institute for Problems in Mechanical Engineering, Russian Academy of Sciences, St. Petersburg 199178, Russia \\ Received: September 08, 2020

\begin{abstract}
Abrief review of the theoretical models which describe specific mechanisms of deformation twinning near crack tips in nanocrystalline and ultrafine-grained materials is presented. In the first model, formation of deformation twins occurs through emission of twinning dislocations from grain boundaries driven by the external stress concentrated by the pre-existent crack. Within the second model, deformation twins are formed due to ideal nanoshear events initiated by high local stresses in the vicinities of crack tips in nanocrystalline materials. These special mechanisms of deformation twinning near crack tips serve as effective toughening micromechanisms in nanostructured materials.
\end{abstract}

\section{INTRODUCTION}

Nanocrystalline and ultrafine-grained materials exhibit the outstanding mechanical properties strongly influenced by their specific structural features, first of all, ultrasmall grain sizes and very large amounts of grain boundaries (GBs) [1-5]. In particular, due to the nanostructure, lattice slip in nanomaterials is either suppressed or shows unusual peculiarities, compared to conventional lattice slip in coarse-grained polycrystals [1-5]. In addition, the nanostructure is responsible for effective operation of specific deformation mechanisms such as deformation twinning. Also, following numerous experimental data, computer simulations and theoretical models, nanoscale twin deformation highly contributes to plastic flow in various nanostructured materials at certain conditions [5-19]. In doing so, twins typically are generated at GBs due to consequent or cooperative emission of partial dislocations from these GBs at comparatively high values of the external stresses [815]. However, formation of nanoscale twins at high stresses can be realized through either consequent or simultaneously occurring ideal nanoshear events $[16,17]$ which serve as an effective alternative to conventional twin formation mechanism [8-15] in nanocrystalline materials. Thus, one of important factors that favor en- hanced deformation twinning in nanocrystalline materials represents operation of very high stresses in such materials during plastic deformation. In this context, it is interesting to understand and describe formation of nanoscale deformation twins in areas near crack tips, where high local stresses operate in nanocrystalline materials. In the first model [18], we consider a conventional mechanism of deformation twin formation occurring through the consequent emission of partial dislocations from a GB in the vicinity of a crack tip in nanostructured materials. In the second model [19], we describe an alternative mechanism of nanoscale deformation twinning which realized through multiplane nanoscale shear events generated at a GB near a crack tip in nanocrystalline materials.

\section{FORMATION OF DEFORMATION TWIN THROUGH SUBSEQUENT EMISSION PARTIAL DISLOCATION FROM GB IN THE VICINITY OF CRACK TIP}

In paper [18], a two-dimensional model of a nanocrystalline material under an external tensile stress $\sigma$ is considered (Fig. 1). Suppose that there is a planar

Corresponding author: N.V. Skiba, e-mail: nikolay.skiba@gmail.com 


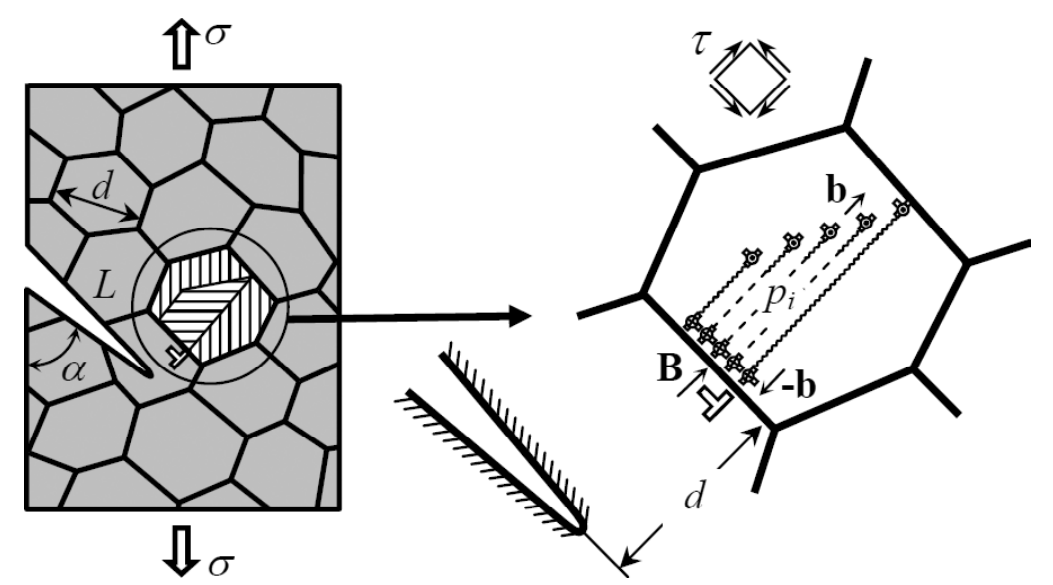

Fig. 1. Formation of a deformation twin at a grain-boundary in the vicinity of a mixed I and II mode crack of length $L$.

mixed (I- and II-) mode crack of length $L$, which is in equilibrium with this stress due to some obstacle. The crack plane is oriented along a plane of maximal shear stress $\tau=\sigma / 2$. Let a perfect lattice edge dislocation with Burgers vector $\boldsymbol{B}$ ( $B$-dislocation) be emitted from the crack tip, glide along an orthogonal plane across a neighboring nanograin and stop at its boundary. Such a dislocation may serve as a source for new lattice dislocations in the next nanograin. As shown in [20], generation of partial dislocations is the most energetically favorable process in this situation. Within the current model adapted for FCC materials, one can expect nucleation of a partial Shockley dislocation with Burgers vector $\boldsymbol{b}$ ( $b$-dislocation) gliding on a (111) plane which is assumed, for the sake of simplicity, to be parallel with the glide plane of the initial $B$-dislocation.

In terms of the continuum approach used here, the nucleation event is considered as appearance of a dipole of partial Shockley dislocations with Burgers vectors $\pm \boldsymbol{b}$. The leading partial ( $b$-dislocation) is supposed to glide into the bulk of the nanograin, while the trailing partial (- $b$-dislocation) rests at the boundary near the $B$ dislocation core. These dislocations have edge components (with Burgers vectors $\pm \boldsymbol{b}_{\mathbf{1}}$ ) and screw components (with Burgers vectors $\pm \boldsymbol{b}_{\mathbf{2}}$ ).

Under the combined action of the external stress concentrated by the crack and stress of the $B$-dislocation, such nucleation events may repeat with producing a number of partial dipoles along neighboring parallel (111) planes (Fig. 1). Gliding along these planes, the emitted $b$-dislocations form stacking-fault strips of width $p_{i}$ ( $i$ is the dislocation number) with a specific surface energy $\gamma$. The stacking-fault strips overlap and produce a twin lamella [21]. If the $b$-dislocation number $i$ exceeds 2 , then glide of this dislocation a distance $p_{i}$ eliminates the stacking fault behind the previous $(i-1)$ th $b$-dislocation until this $i$ th $b$-dislocation passes ahead of the $(i$ 2 )th $b$-dislocation [21]. In Fig. 1, where this situation is illustrated for five emitted $b$-dislocations, the dashed lines mark "healed" regions with eliminated stacking faults. As a result of this healing, the total stacking fault energy of the growing twin lamella remains the same, whereas glide of $b$-dislocations provides a plastic relaxation and diminishes the total energy of the system. We guess this is one of the main causes of the cooperative glide of twinning $b$-dislocations on neighboring crystallographic planes.

In the model under consideration, the emission of the first $b$-dislocation becomes possible when the external shear stress $\tau$ achieves a certain critical value $\tau_{c}^{(1)}$. At $\tau \geq \tau_{c}^{(1)}$, the first $b$-dislocation is emitted from the grain boundary and, depending on the model parameters, either reaches the opposite grain boundary or occupies a certain equilibrium position inside the grain. In any case, this dislocation hinders the emission of the next $b$-dislocation, which may be emitted only if the external shear stress $\tau$ increases to a new critical value $\tau_{c}^{(2)}>\tau_{c}^{(1)}$. After its emission, the second $b$-dislocation occupies a certain equilibrium position between the point of emission and the first $b$-dislocation due to the repulsive interaction of these two dislocations. In its turn, the first $b$ dislocation is shifted ahead to a new equilibrium position. This situation is repeated during the emission of the next $b$-dislocations. Every $b$-dislocation emission requires a further increase in the critical stress $\tau_{c}^{(n)}>\tau_{c}^{(n-1)}$ and moves the $b$-dislocations emitted earlier toward to new equilibrium positions. During this process, some of the $b$-dislocations may reach the opposite grain boundary. As a result of this sequential emission of $b$-dislocations and the overlap of the stacking-fault trips behind them, a rather thick twin lamella may form (Fig. 1).

In terms of our model, a deformation twin forms via the sequential emission of twinning $(b-)$ dislocations from the region near an extrinsic grain-boundary $(B-)$ dislocation under the combined action of an external 
shear stress (concentrated by a crack) and the dislocation stress field (Fig. 1). Consider the conditions which are necessary for the emission of any of the twinning dislocations. In doing so, we study the transition of the defect system from the $(n-1)$ th state (here $n>1)$ with a total energy $W_{n-1}$, which corresponds to the presence of $(n-1)$ dipoles of partial $\pm b$-dislocations, into the $n$th state with a total energy $W_{n}$, which corresponds to the generation of an $n$th dislocation dipole. This transformation of the defect structure is energetically favorable if $\Delta W_{n}=W_{n}-W_{n-1}<0$. From the condition $\Delta W_{n}=0$, we then find the critical stress $\tau_{c}^{(n)}$ of the transition of the defect system from the $(n-1)$ th state into the $n$th state. The case of $n=1$ is analyzed in a similar way with $\Delta W_{1}=W_{1}$.

In the $(n-1)$ th state, when $n>1$, the total energy of the defect system is given by

$$
W_{n-1}=E_{s e l f \Sigma}^{b(n-1)}+E_{\mathrm{int} \Sigma}^{C-b(n-1)}+E_{\mathrm{in} \Sigma}^{B-b(n-1)}+E_{\mathrm{int} \Sigma}^{b-b(n-1)}+E_{\gamma \Sigma}^{(n-1)}+E_{\tau \Sigma}^{(n-1)},
$$

where we omit the terms which do not depend on the number $n$ - 1 of emitted $b$-dislocations. Here $E_{\text {self } \Sigma}^{b(n-1)}$ is the sum self-energy of $n$-1 dipoles of partial $\pm b$-dislocations, $E_{i n t \Sigma}^{C-b(n-1)}$ is the sum energy of elastic interaction between the crack and all these dipoles, $E_{i n t \Sigma}^{B-b(n-1)}$ is the sum energy of elastic interaction between the $B$-dislocation and all these dipoles, $E_{i n t \Sigma}^{b-b(n-1)}$ is the sum energy of elastic interaction between all these dipoles, $E_{\gamma \Sigma}^{(n-1)}$ is the total energy of all retained stacking-fault segments, and $E_{\tau \Sigma}^{(n-1)}$ is the total interaction energy of the external shear stress $\tau$ with all $n-1$ partial $b$-dislocations. It is worth to note that introducing the terms $E_{i n t \Sigma}^{C-b(n-1)}$ and $E_{\tau \Sigma}^{(n-1)}$, we separately consider the "background" stress level $\tau$ and its perturbation $\sigma_{x y}^{c}$ caused by the crack, although their sum, $\tau+\sigma_{x y}^{c}$, acts on partial $b$ dislocations in reality. Also, in calculating the energy terms $E_{s e l f \Sigma}^{b(n-1)}, E_{i n t \Sigma}^{B-b(n-1)}$, and $E_{i n t \Sigma}^{b-b(n-1)}$ figuring in Eq. (1), we will neglect the screening influence of the crack free surfaces on the dislocation stress fields. It seems to be reasonable in this situation (Fig. 1) when a twin lamella is generated so far from the crack tip that the lamella sizes are not larger than its distance from the crack tip and surface.

The total self-energy of $n$ - 1 dipoles of partial $\pm b$-dislocations is the sum of their self-energies as follows

$$
E_{\text {self } \Sigma}^{b(n-1)}=D \sum_{i=1}^{n-1}\left\{b_{1}^{2}\left(\ln \frac{p_{i}-r_{c 1}}{r_{c 1}}+1\right)+(1-v) b_{2}^{2}\left(\ln \frac{p_{i}-r_{c 2}}{r_{c 2}}+1\right)\right\},
$$

where $D=G /[2 \pi(1-v)], G$ is the shear modulus, $v$ is the Poisson ratio, $p_{i}$ is the path of the $i$ th $b$-dislocation, $\quad r_{c 1} \approx$ $b_{1}$ and $r_{c 2} \approx b_{2}$ are the cut-off radii of the stress fields of the edge and screw components of $b$-dislocations, respectively. The units in the brackets approximate the contribution of the dislocation cores [21].

The total energy of elastic interaction between the crack and all $n$ - 1 dipoles of partial $\pm b$-dislocations is calculated as a work spent by the crack to nucleate these dipoles in the crack stress $\sigma_{x y}^{c}$ :

$$
E_{\mathrm{int} \Sigma}^{C-b(n-1)}=b_{1} \sum_{i=1}^{n-1} \int_{r_{c 1}}^{p_{i}-r_{c 1}} \sigma_{x y}^{C}\left(x, y=y_{i}\right) \mathrm{d} x
$$

where $\left(0, y_{i}\right)$ is the generation point for the $i$ th dislocation dipole (Fig. 1). The crack stress $\sigma_{x y}^{c}$ is calculated in Appendix A of work [18].

The total energy of elastic interaction between the $B$-dislocation and all $n$ - 1 dipoles of partial $\pm b$-dislocations is found in a similar way that gives

$$
E_{\mathrm{int} \Sigma}^{B-b(n-1)}=\frac{D B b_{1}}{2} \sum_{i=1}^{n-1}\left(\ln \frac{y_{i}^{2}}{y_{i}^{2}+p_{i}^{2}}-\frac{y_{i}^{2}}{y_{i}^{2}+p_{i}^{2}}+1\right) .
$$

The elastic interaction energy between the $i$ th and $j$ th dipoles of partial $\pm b$-dislocations is calculated by considering the generation of an ith dipole in the shear-stress field of the jth dipole (see, e.g., [18,19]). The energy is the total energy of all such pair interactions between dislocation dipoles and can be written in the form of a double sum over subscripts $i$ and $j$

$$
\begin{aligned}
& E_{\mathrm{int} \Sigma}^{b-b(n-1)}=\frac{D b_{1}^{2}}{2} \sum_{i=1}^{n-2} \sum_{j=i+1}^{n-1}\left\{2 y_{i j}^{2}\left(\frac{p_{i}^{2}-2 p_{i} p_{j}}{\left(p_{j}^{2}+y_{i j}^{2}\right)\left(p_{j}^{2}+p_{i}^{2}+y_{i j}^{2}-2 p_{i} p_{j}\right)}-\frac{p_{i}^{2}}{y_{i j}^{2}\left(p_{j}^{2}+y_{i j}^{2}\right)}\right)+\right. \\
& \left.\ln \left[1+\frac{p_{i}^{2}}{y_{i j}^{2}}\right]-\ln \left[1+\frac{p_{i}^{2}-2 p_{i} p_{j}}{p_{j}^{2}+y_{i j}^{2}}\right]\right\},
\end{aligned}
$$



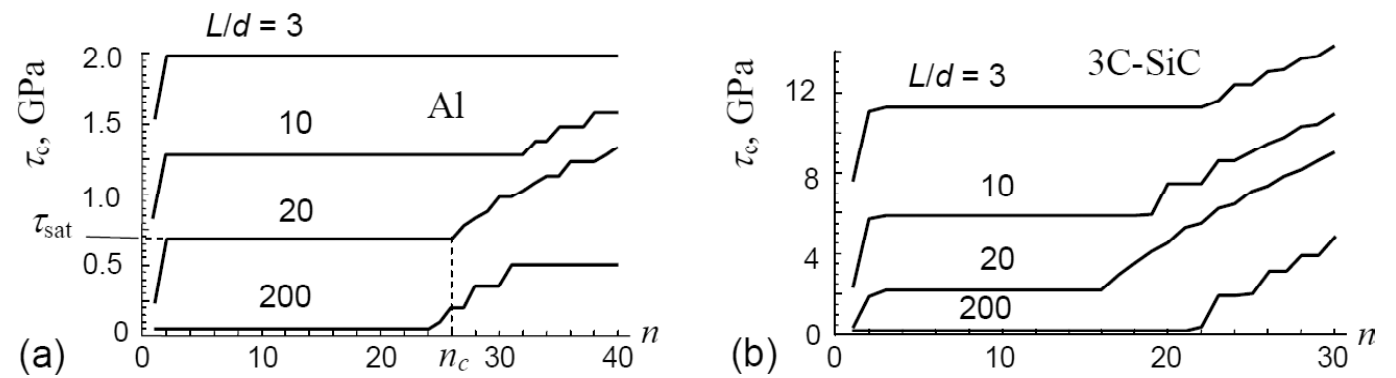

Fig. 2. Dependence of the critical shear stress $\tau_{c}$ for the emission of the $n$-th partial dislocation in nanocrystalline (a) $\mathrm{Al}$ and (b) 3C-SiC, for various values of the crack length: $L=3 d, 10 d$, and $20 d$.

with $y_{i j}=y_{j}-y_{i}$

The total energy of all stacking-fault strips (or their retained segments) is

$$
E_{\gamma \sqrt{\Sigma}}^{(n-1)}=\left\{\begin{array}{l}
\gamma p_{1}, n-1=1 \\
\gamma\left(p_{1}+p_{2}\right), n-1 \geq 2
\end{array} .\right.
$$

The total interaction energy of the external shear stress $\tau$ with all $n$-1 partial $b$-dislocations reads

$$
E_{\tau \Sigma}^{(n-1)}=-b_{1} \tau \sum_{i=1}^{n-1} p_{i}
$$

The total energy of the defect system in the $n$th state can be written as

$$
\begin{aligned}
& W_{n}=E_{s e l f \Sigma}^{b(n)}+E_{\mathrm{int} \Sigma}^{C-b(n)}+E_{\mathrm{int} \Sigma}^{B-b(n)}+ \\
& E_{\mathrm{int} \Sigma}^{b-b(n)}+E_{\gamma \Sigma}^{(n)}+E_{\tau \Sigma}^{(n)} .
\end{aligned}
$$

The calculation of the energy terms entering into Eq. (8) is completely analogous to the calculation of the respective energies in Eq. (1) if we substitute $n$-1 for $n$ (where $n>1$ ) and determine new stable equilibrium positions $p_{i}$ of the previously emitted partial $b$-dislocations.

In the case of $n=1$, the total energy $W_{1}$ is determined by Eq. (8) with $n=1$ and $E_{i n c}^{b-b(n-1)}=0$.

Thus, we determined all the terms in the expressions for the energies $W_{n-1}$ and $W_{n}$ involved in the difference $\Delta W_{n}=W_{n}-W_{n-1}$.

The stable equilibrium positions $\tilde{p}_{i}$ of the previously emitted $b$-dislocations correspond to minima in the $\Delta W_{n}\left(p_{i}\right)$ dependences and can be found from the equation $\partial \Delta W_{n} / \partial p_{i}=0$. Using the equation $\Delta W_{n}=0$, we can determine the critical shear stress $\tau_{c}^{(n)}$ for the generation of an $n$th dipole of partial $\pm b$-dislocations. We employed this equation and the aforementioned algorithm of calculating the stable equilibrium positions of emitted $b$-dislocations to find the dependences of the critical stress $\tau_{c}$ on the number of partial dislocations $n$ at various lengths $L$ of the crack. We performed calculations for nanocrystalline $\mathrm{Al}$ and $3 \mathrm{C}-\mathrm{SiC}$ using the following model parameter values: for $\mathrm{Al}, G=27 \mathrm{GPa}, v=0.31$ [21], $\gamma=120 \mathrm{~mJ} \mathrm{~m}^{-2}$ [22], $b_{1} \approx 0.143 \mathrm{~nm}$ and $b_{2} \approx 0.022 \mathrm{~nm}$ [21]; for $3 \mathrm{C}-\mathrm{SiC}, G=217 \mathrm{GPa}, \nu=0.23$ [23], $\gamma=0.1 \mathrm{~mJ} \mathrm{~m}^{-2}$ [24], $b_{1} \approx 0.154 \mathrm{~nm}$ and $b_{2} \approx 0.024 \mathrm{~nm}$ [24]. The results are shown in Figs. $2 \mathrm{a}$ (for $\mathrm{Al}$ ) and $2 \mathrm{~b}$ (for $3 \mathrm{C}-\mathrm{SiC}$ ) at the crack lengths $L=3 d, 10 d$, and $20 d$, where $d=30 \mathrm{~nm}$ is the mean grain size and the distance between the crack tip and the $B$-dislocation. In all cases, the critical shear stress $\tau_{c}$ is seen to increase at the initial stage of twin formation (the first stage of local strengthening). After reaching a certain value $\tau_{\text {sar }}$, the quantity $\tau_{\mathrm{c}}(n)$ becomes independent of the number of emitted twinning dislocations $n$ till a certain number $n_{c}$ (the stage of local flow due to the thickening of the twin lamella). Finally, for a twinning dislocation whose number exceeds $n_{c}$ to be emitted, the critical shear stress should again increase (the second stage of local strengthening). As follows from the dependences shown in Fig. 2, an increase in the crack length in both $\mathrm{Al}$ and $3 \mathrm{C}-\mathrm{SiC}$ leads to a substantial decrease in the critical stress.

In doing aforementioned calculations, we always convinced that, for the chosen values of the model parameters (the external shear stress $\tau$ and the crack length $L$ ), the deformation-twin formation was energetically favorable and passed without overcoming any energy barriers.

\section{FORMATION DEFORMATION TWINS THROUGH IDEAL NANOSHEAR EVENTS NEAR CRACK TIP}

In paper [19], the mode I crack of length $l$ concentrates the external stress near its tip, and the resulting local stress induces generation of a deformation twin $A B C D$ (Figs. 3a and 3b). Following the approach [17], the deformation twin near the crack tip is generated and evolves through the events of ideal nanoscale shear consequently occurring on parallel glide planes, as it is schematically shown in Fig. 3. In terms of dislocations, the twin formation process (Fig. 3) is represented as consequent generation of $n$ dislocation dipoles on parallel glide planes. In doing so, generation of each dislo- 
(a)

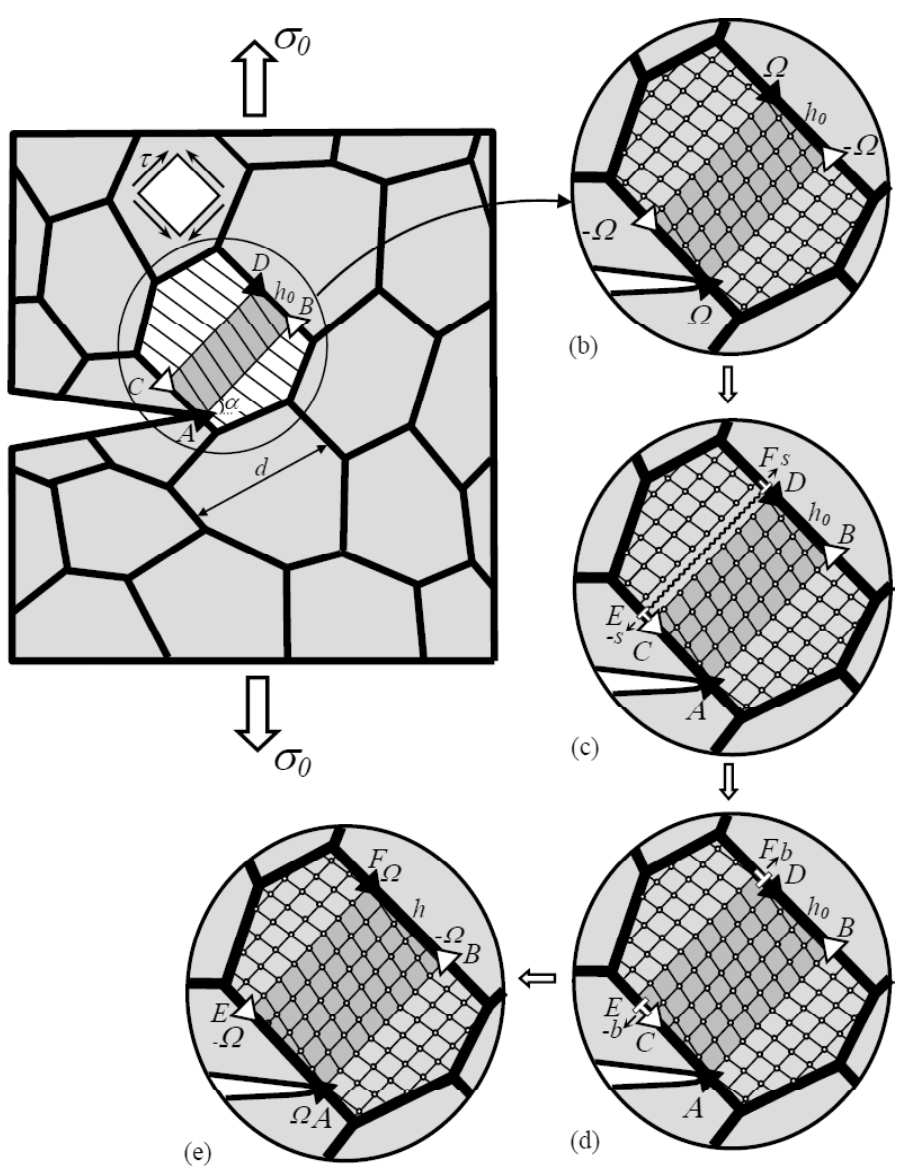

Fig. 3. A two-dimensional model of nanotwin formation near a crack tip. (a,b) An initial defect configuration with a crack and a nanotwin $A B C D$. (c) Formation of a dipole $E F$ of partial non-crystallographic dislocations with the growing Burgers vector magnitude $s$. (d) The Burgers vector magnitude $s$ of the dipole dislocation gradually increases and reaches the magnitude $b$ of the Burgers vector of a partial dislocation. (e) As a result, the length of the nanotwin $A B C D$ increases, and the nanotwin $A B C D$ transforms into the nanotwin $A B E F$.

cation dipole is realized through both growth of the magnitude $s$ of the Burgers vectors of its dislocations from 0 to $b$ (with $b$ being the magnitude of the Shockley partial) and corresponding evolution of generalized stacking faults located between immobile dislocations composing the dipole. The nanoscale twin $A B C D$ (Fig. $3 a)$ resulted from $n$ ideal nanoshear events creates internal stresses in the specimen. Following the approach [17], the twin $A B C D$ (Fig. 3a) as a stress source can be effectively represented as a quadrupole of wedge disclinations characterized by strengths $\pm \Omega$ (hereinafter called $\pm \Omega$-disclinations) (Fig. $3 b$ ). In doing so, according to the theory of disclinations, the disclination strength magnitude $\mathrm{W}$ is in the following relationship with $b$ and the distance $p$ between neighboring dislocation dipoles: $\Omega=2 \arctan (\mathrm{b} / 2 p)$. The nanoscale twin $A B C D$ is supposed to have a rectangular shape with the sizes $d$ and $h_{0}$ (Fig. 3a,b) called also the disclination quadrupole arms. The distance $p$ between the neighboring dipoles of $\pm s$-dislocations is equal to the distance between the $\{111\}$ crystal planes and related to the crystal lattice parameter $a$ by the equality $p=a / \sqrt{3}$. Within our model, the examined Shockley partials are edge dislocations with the Burgers vectors of the $(\mathrm{a} / 6)<11 \overline{2}>$ type. The magnitude $b$ of such Burgers vectors is equal to $a / \sqrt{6}$.

Let the nanotwin $A B C D$ consisting of dipoles of Shockley partials grow through the formation of $(n+1)$ st dipole $E F$ of Shockley partials adjacent to the preexistent nanotwin near a crack tip (Fig. 3c). As it has been discussed above, we assume that the dipole $E F$ of Shockley partials forms via the growth of the magnitude $s$ of the Burgers vectors of its dislocations from 0 to $b$ and corresponding evolution of generalized stacking faults located between these immobile dislocations composing the dipole (Figs. 3c and 3d). As a result, the length $h_{0}$ of the nanotwin $A B C D$ increases by $p$ (the distance between the $\{111\}$ crystal planes). In other words, the new nanotwin $A B E F$ forms which has the length $h=h_{0}+p$ (Fig. 3e). Within our model, the above process of the nanotwin growth repeats until its further growth stops to be energetically favored. In order to 
estimate the conditions for energetically favorable nanotwin growth near a crack tip, in the following section, we will calculate the energy characteristics of the nanotwin growth (Fig. 3).

Further, calculate the energy change due to the generation of a dipole $E F$ of the partial $\pm s$-dislocations (Figs. $3 \mathrm{c}-3 \mathrm{e}$ ) in a nanocrystalline solid containing a crack and a pre-existent nanotwin $A B C D$. The energy change $\triangle W$ (per dislocation unit length) can be presented as

$$
\Delta W=E^{s}+E^{s-q}+E^{s-\sigma}+E_{\gamma},
$$

where $E^{s}$ denotes the self-energy of the dipole of ${ }_{s}$ dislocations, $E^{s-q}$ designates the energy of the interaction between the stress fields of the dipole $E F$ of the $\pm s$ dislocations and the quadrupole $A B C D$ of wedge disclinations with the strengths $\pm \Omega, E^{s-q}$ specifies the energy of the interaction of the dipole $E F$ of the $\pm_{S-}$ dislocations with the stress field $\sigma_{i j}^{c}$ created by the applied load $\sigma_{0}$ in the solid with a crack, and $E_{\gamma}$ is the energy of the generalized stacking fault that joins the dipole dislocations.

In a first approximation, we neglect the effect of the crack on the energy $E^{s}$. Then the energy $E^{s}$ is calculated as the energy of a dislocation dipole in an isotropic solid [21]:

$$
E^{s}=D s^{2}\left(\ln \frac{d}{r_{c}}+1\right),
$$

where $D=G /[2 \pi(1-v)], G$ is the shear modulus, $v$ is Poisson's ratio, and $r_{c} \approx s$ is the dislocation core cutoff radius.

The energy $E^{s-q}$ of the elastic interaction between the disclination quadrupole and the dipole of $\pm s$-dislocations can be calculated as the work spent to the generation of the dislocation dipole in the stress field of the disclination quadrupole [25]. For the calculation of the energy $E^{s-q}$ we will also neglect the presence of the crack. Then the final expression for the energy $E^{s-q}$ follows as:

$$
E^{s-q}=D \Omega b\left(h \ln \frac{d^{2}+h^{2}}{h^{2}}-p \ln \frac{d^{2}+p^{2}}{p^{2}}\right) .
$$

The energy $E^{s-q}$ can be written as [18]:

$$
E^{s-\sigma}=-b \int_{0}^{d} \sigma_{x y}^{c} \mathrm{~d} x
$$

where $\sigma_{x y}^{c}$ is the component of the stress field $\sigma_{i j}^{c}$ (see, e.g., [18]) created by the applied tensile load $\sigma_{0}$ in the solid with a flat crack of length $l$.

The energy $E_{\gamma}$ of the generalized stacking fault that joins the opposite dislocations with the Burgers vec- tors $\pm \mathbf{S}$ and is adjacent to $n$ stacking faults that compose the nanotwin $A B C D$ is given [26,27] by

$$
E_{\gamma}=\gamma_{n+1}(s) d,
$$

where

$$
\gamma_{n}(s)=\left(\gamma_{u t}-2 \gamma_{t s f}\right) \sin ^{2}(\pi s / b), n \geq 3,
$$

$\gamma_{1}(s)=\left\{\begin{array}{l}\gamma_{u s} \sin ^{2}(\pi s / b), 0 \leq s / b \leq 1 / 2, \\ \gamma_{i s f}+\left(\gamma_{u s}-\gamma_{i s f}\right) \sin ^{2}(\pi s / b), 1 / 2<s / b \leq 1,\end{array}\right.$

$\gamma_{2}(s)=\left\{\begin{array}{l}\left(\gamma_{u t}-\gamma_{i s f}\right) \sin ^{2}(\pi s / b), 0 \leq s / b \leq 1 / 2, \\ \left(\gamma_{u t}-2 \gamma_{t s f}\right) \sin ^{2}(\pi s / b), 1 / 2<s / b \leq 1,\end{array}\right.$

with $\gamma_{u t}, \gamma_{t s}, \gamma_{u s}$ and $\gamma_{i s f}$ being the material constants.

Now the characteristic energy change $\Delta W$ is given by formulae (9) to (16). Let is calculate the dependence $\Delta W(s / b)$ in the exemplary case of nanocrystalline Ni. For $\mathrm{Ni}$, we use the following parameter values: $G=73 \mathrm{GPa}$, $v=0.34, a=352 \mathrm{~nm}[21], \gamma_{u t}=0.324 \mathrm{~J} / \mathrm{m}^{2}, \gamma_{t s f}=0.055 \mathrm{~J} / \mathrm{m}^{2}$, $\gamma_{u s}=0.273 \mathrm{~J} / \mathrm{m}^{2}$ and $\gamma_{i s f}=0.110 \mathrm{~J} / \mathrm{m}^{2}$ [26]. The dependences $\mathrm{D} W(s / b)$ for nanocrystalline $\mathrm{Ni}$ are plotted in Fig. 4, for $d=20 \mathrm{~nm}, l=100 \mathrm{~nm}, n=10, \alpha=70^{\circ}$, and various values of the applied shear stress $\tau$. (The applied shear stress $\tau$ is defined here as the shear stress that would be created by the applied tensile load $\sigma_{0}$ in the crack plane in the absence of this crack, that is, $\tau=\sigma_{0} \sin \alpha \cos \alpha$.) As it follows from Fig. 4, at $\tau=0.5 \mathrm{GPa}$ or $1 \mathrm{GPa}$, the energy change $\Delta W$ is not a monotonously decreasing function of $s$ being in the range $0<s / b<1$. That is, at these values of the shear stress $\tau$, the formation of a new dipole of the Shockley partials (with $s / b=1$ ) requires surmounting an energy barrier. In contrast, at larger values of $\tau(1.5 \mathrm{GPa}$ or $2 \mathrm{GPa}), \Delta W$ monotonously decreases with an increase in being in the range $0<s / b<1$. This implies that in the discussed situation, the formation of a new dipole of

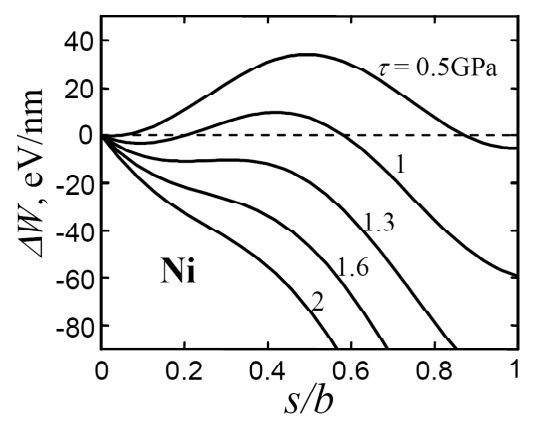

Fig. 4. Dependences of the energy change $\Delta W$ on the normalized magnitude $s / b$ of the dipole dislocation Burgers vector, for various values of the applied shear stress $\tau$. 
Shockley partials (necessary for the growth of the nanotwin $A B C D$ ) does not require overcoming an energy barrier. The analysis demonstrates that for the parameter values used to plot the curves in Fig. 4, the nonbarrier nanotwin growth is realized at shear stresses $\tau$ exceeding 1.2 GPa.

\section{CONCLUSIONS}

Thus, specific mechanisms for formation of nanoscale deformation twins near crack tips in nanocrystalline and ultrafine-grained materials were theoretically described. These twin formation mechanisms near crack tips represent: the consequent events of partial dislocation emission from GBs and the nanoscale shear events at GBs. The energy and stress characteristics of the nanoscale twin formation through these special mechanisms in nanocrystalline aluminum $(\mathrm{Al})$, silicon carbide $(\mathrm{SiC})$ and nickel (Ni) were calculated. It was shown that the deformation twinning mechanisms can effectively operate near crack tips in nanocrystalline and ultrafine-grained materials at rather high, but realistic levels of the stress. Also, it was demonstrated that the specific mechanisms of deformation nanotwinning in the vicinities of crack tips serve as an effective toughening micromechanisms releasing in part local stresses near crack tips in nanostructured materials.

\section{REFERENCES}

[1] M.A. Meyers, A. Misra and D.J. Benson, Mechanical properties of nanocrystalline materials, Progr. Mater. Sci., 2006, vol. 51, no. 4, pp. 427-556. https://doi.org/10.1016/ j.pmatsci.2005.08.003

[2] M. Dao, L. Lu, R.J. Asaro, J.T.M. De Hosson and E. Ma, Toward a quantitative understanding of mechanical behavior of nanocrystalline metals, Acta Mater., 2007, vol. 55, no. 12, pp. 4041-4065. https://doi.org/10.1016/j.actamat.2007.01.038

[3] C.C. Koch, Structural nanocrystalline materials: an overview, J. Mater. Sci., 2007, vol. 42, pp. 1403-1414. https://doi.org/10.1007/s10853-0060609-3

[4] C.S. Pande and K.P. Cooper, Nanomechanics of Hall-Petch relationship in nanocrystalline materials, Progr. Mater. Sci., 2009, vol. 54, no. 6, pp. 689-706. https://doi.org/10.1016/ j.pmatsci.2009.03.008

[5] Y.T. Zhu, X.Z. Liao and X.L. Wu, Deformation twinning in nanocrystalline materials, Progr. Mater. Sci., 2012, vol. 57, no. 1, pp. 1-62. https://doi.org/10.1016/j.pmatsci.2011.05.001.
[6] H. Abdolvand, K. Louca, C. Mareau, M. Majkut and J. Wright, On the nucleation of deformation twins at the early stages of plasticity, Acta Materialia, 2020, vol. 196, pp. 733-746. https://doi.org/10.1016/j.actamat.2020.07.010

[7] R. Sidharth, W. Abuzaid and H. Sehitoglu, Nanotwinning enhanced room temperature fatigue crack growth in single crystalline CoCrFeMnNi high entropy alloy, Intermetallics, 2020, vol. 126, art. 106919. https://doi.org/10.1016/ j.intermet.2020.106919

[7] T. He and M. Feng, Effect of nanotwin near a branched crack tip on crack blunting in deformed nanocrystalline materials, Acta Mechanica, 2018, vol. 229, pp. 3223-3234. https://doi.org/10.1007/s00707-018-2158-7

[8] Z.Y. Liang, J.T.M. De Hosson and M.X. Huang, Size effect on deformation twinning in face-centred cubic single crystals: Experiments and modelling, Acta Materialia, 2017, vol. 129, pp. 1-10. https://doi.org/10.1016/j.actamat.2017.02.063

[9] X.Z. Liao, F. Zhou, E.J. Lavernia, D.W. He and Y.T. Zhu, Deformation twins in nanocrystalline Al, Appl. Phys. Lett., 2003, vol. 83, art. 5062. https://doi.org/10.1063/1.1633975

[10] M. Chen, E. Ma, K.J. Hemker, H. Sheng, Y. Wang and X. Cheng, Deformation twinning in nanocrystalline aluminum, Science, vol. 300, no. 5623, pp. 1275-1277. https://doi.org/10.1126/ science. 1083727

[11] X.Z. Liao, Y.H. Zhao, S.G. Srinivasan, Y.T. Zhu, R.Z. Valiev and D.V. Gunderov, Deformation twinning in nanocrystalline copper at room temperature and low strain rate, Appl. Phys. Lett., 2004, vol. 84, no. 4, pp. 592-594. https://doi.org/10.1063/1.1644051

[12] Y.M. Wang, A.M. Hodge, J. Biener, A. V. Hamza, D.E. Barnes, K. Liu and T.G. Nieh, Deformation twinning during nanoindentation of nanocrystalline Ta, Appl. Phys. Lett., 2005, vol.86, art. 201915. https://doi.org/10.1063/ 1.1883335

[13] M.Y. Gutkin, I.A. Ovid'ko and N.V. Skiba, Generation of deformation twins in nanocrystalline metals: Theoretical model, Phys. Rev. B, 2006, vol.74, art. 172107. https://doi.org/10.1103/PhysRevB.74.172107

[14] I.A. Ovid'ko and N.V. Skiba, Nanotwins induced by grain boundary deformation processes in nanomaterial, Scripta Materialia, 2014, vol. 71, pp. 33-36. https://doi.org/10.1016/ j.scriptamat.2013.09.028 
[15] I.A. Ovid'ko and N.V. Skiba, Generation of nanoscale deformation twins at locally distorted grain boundaries in nanomaterials, Int. J. Plasticity, 2014, vol. 62, pp. 50-71. https://doi.org/ 10.1016/j.ijplas.2014.06.005

[16] I.A. Ovid'ko, Nanoscale multiplane shear and twin deformation in nanowires and nanocrystalline solids, Appl. Phys. Lett., 2011, vol. 99, art. 061907. https://doi.org/10.1063/ 1.3620934

[17] I.A. Ovid'ko and A.G. Sheinerman, Deformation Twinning through Nanoscale Ideal Shears in Nano- and Polycrystalline Materials at Ultra High Stresses, Rev. Adv. Mater. Sci., 2011, vol. 27, no. 2, pp. 189-194. http://www.ipme.ru/e-journals/ RAMS/no_22711/ovidko.pdf

[18] M.Y. Gutkin, I.A. Ovid'ko and N.V. Skiba, Crackstimulated generation of deformation twins in nanocrystalline metals and ceramics, Phil. Mag., 2008, vol. 88, no. 8, pp. 1137-1151. https://doi.org/ 10.1080/14786430802070813

[19] N.F. Morozov, I.A. Ovid'ko, A.G. Sheinerman and N.V. Skiba, Formation of Deformation Twins through Ideal Nanoshear Events near Crack Tips in Deformed Nanocrystalline Materials, Rev. Adv. Mater. Sci., 2012, vol. 32, no. 1, pp. 7581. http://www.ipme.ru/e-journals/RAMS/ no_13212/12_13212_morozov.pdf

[20] M.Yu. Gutkin and I.A. Ovid'ko, Generation of dislocation loops in deformed nanocrystalline materials, Phil. Mag., 2006, vol. 86, no. 11, pp. 1483-1511. https://doi.org/10.1080/ 14786430500199302
[21] J.P. Hirth and J. Lothe, Theory of dislocations (Wiley, New York, 1982).

[22] R.J. Asaro and S. Suresh, Mechanistic models for the activation volume and rate sensitivity in metals with nanocrystalline grains and nanoscale twins, Acta Mater., 2003, vol. 53. no. 12, pp. 3369-3382. https://doi.org/10.1016/ j.actamat.2005.03.047

[23] Z. Ding, S. Zhou and Y. Zhao, Hardness and fracture toughness of brittle materials: A density functional theory study, Phys. Rev. B, 2007, vol. 70, art. 184117. https://doi.org/10.1103/ PhysRevB.70.184117

[24] U. Kaiser and I.I. Khodos, On the determination of partial dislocation Burgers vectors in fee lattices and its application to cubic SiC films, Phil. Mag. A, 2002, vol. 82, no. 3, pp. 541-551. https://doi.org/10.1080/01418610208239615

[25] A.E. Romanov and A.L. Kolesnikova, Application of disclination concept to solid structures, Progr. Mater. Sci., 2009, vol.54, no. 6, pp. 740-769. https://doi.org/10.1016/j.pmatsci.2009.03.002

[26] S. Kibey, J.B. Liu, D.D. Johnson and H. Sehitoglu, Predicting twinning stress in fcc metals: Linking twin-energy pathways to twin nucleation, Acta Mater., 2007, vol. 55, no. 20, pp. 6843-6851. https://doi.org/10.1016/j.actamat.2007.08.042

[27] S.V. Bobylev and I.A. Ovid'ko, Nanodisturbances and nanoscale deformation twins in $f_{c c}$ nanowires, Phys. Rev. B, 2011, vol. 83, art. 054111. https://doi.org/10.1103/

PhysRevB.83.054111 